\title{
Incident Direction Independent Wave Propagation and Unidirectional Lasing
}

\author{
L. Jin ${ }^{*}$ and Z. Song \\ School of Physics, Nankai University, Tianjin 300071, China
}

(Received 2 April 2018; published 15 August 2018)

\begin{abstract}
We propose an incident direction independent wave propagation generated by properly assembling different unidirectional destructive interferences (UDIs), which is a consequence of the appropriate match between synthetic magnetic fluxes and the incident wave vector. Single-direction lasing at spectral singularity is feasible without introducing nonlinearity. UDI allows unidirectional lasing and unidirectional perfect absorption; when they are combined in a parity-time-symmetric manner, the spectral singularities vanish with bounded reflections and transmissions. Furthermore, the simultaneous unidirectional lasing and perfect absorption for incidences from opposite directions is created. Our findings provide insights into light control and may shed light on the explorations of desirable functionality in fundamental research and practical applications.
\end{abstract}

DOI: 10.1103/PhysRevLett.121.073901

Introduction.-Parity-time $(\mathcal{P} \mathcal{T})$ symmetry has been theoretically and experimentally investigated in a variety of non-Hermitian systems [1-22]; non-Hermiticity controls the exact and broken $\mathcal{P} \mathcal{T}$-symmetric phases [18-22]. The phase transition points are exceptional points [23-27] utilized for sensing enhancement [28-32]. The topologies of exceptional points are distinct [33-37]. Spectral singularities (SSs) in scattering systems belong to another type of non-Hermitian singularities, at which eigenstate completeness is spoiled [38,39]; incident waves from opposite directions at an appropriate phase match are perfectly absorbed in a coherent perfect absorber [40-48].

Non-Hermitian character causes unidirectionality [49-54], the fundamental mechanism of which differs from that created by chiral light-matter interaction [55-59]. Typical phenomena include unidirectional reflectionlessness $[49,50]$ and unidirectional spectral singularity that allows unidirectional perfect absorption (UPA) and unidirectional lasing (UL) [51,52]; however, the transmissions there protected by symmetry are reciprocal [60-63]. Nonreciprocal transmission is indispensable for optical information processing. Nonreciprocity, implemented via magneto-optic effect [64] and optical nonlinearity [65], has been created based on various strategies in linear and magnetic-free devices [66,67], in singlephoton level [68,69], and even in acoustics [70]. Benefited from synthetic magnetic flux realized for photons [71-79] and progress in non-Hermitian physics [22], non-Hermiticity associated with synthetic magnetic flux

Published by the American Physical Society under the terms of the Creative Commons Attribution 4.0 International license. Further distribution of this work must maintain attribution to the author(s) and the published article's title, journal citation, and DOI. induces nonreciprocal transmission in linear photonic lattices [80-82].

In this Letter, we propose an incident direction independent wave propagation, which is an extraordinary asymmetry in both reflectivity and transmittivity that stemmed from the unidirectional destructive interference (UDI); single-direction lasing occurs at the SS, where an incidence from either side induces a UL toward the same direction. The appropriate match between synthetic magnetic fluxes in different functional UDIs creates many intriguing unidirectional phenomena beyond one-way propagation, UPA, and UL [82]. With judiciously chosen synthetic magnetic fluxes, detunings, and the gain or loss of side-coupled resonators, the SS exhibits UPA or UL; their coincidence leads to an SS elimination under $\mathcal{P} \mathcal{T}$ symmetry. Furthermore, a simultaneous unidirectional lasing and perfect absorption for incidences from opposite sides is feasible by assembling different unidirectional elements. These novel wave propagation phenomena facilitate various applications of non-Hermitian metamaterials without introducing nonlinearity [83-86].

Model.-We consider a one-dimensional uniformly coupled passive resonator chain in Fig. 1(a) with identical resonant frequency $\omega_{c}$; the primary resonators (roundshaped) are evanescently coupled through the auxiliary resonators (stadium-shaped), which are antiresonant with the primary resonators. The coupling strength $J=1$ is set to be unity. A resonator is side-coupled to the resonator chain with one asymmetric coupling, which is introduced through the optical path length difference $2 \Delta x$ in the tunneling between resonators $\alpha$ and 0 [76]. An extra direction dependent phase factor $e^{ \pm i \phi_{\alpha}}$ is equivalently induced in the effective coupling between resonators $\alpha$ and 0 , resulting in a synthetic magnetic flux $\phi_{\alpha}=$ $2 \pi \Delta x / \lambda \equiv(-e / \hbar) \oint \vec{A}_{\alpha} \cdot d \vec{l}$ in the triangle that associated 
with a vector potential $\vec{A}_{\alpha}[73,74,79]$, where $\lambda$ is the resonant wavelength [77]. The resonators support the counterclockwise $(\mathrm{CCW})$ and clockwise $(\mathrm{CW})$ modes; they experience opposite synthetic magnetic fluxes. The CCW mode is analyzed without loss of generality, and the CW mode is discussed as well.

The frequency detuning (net gain or loss) of resonator $\alpha$ is represented by the real (imaginary) part of $V_{\alpha}$. The loss is the dissipation $\omega_{c} /\left(2 Q_{\alpha}\right)$ caused by the interaction between resonator $\alpha$ and its environment, where $Q_{\alpha}$ is the quality factor. The gain is induced by pumping the ions doped in the resonator $[14,20]$. Assuming a weak pump that far away from gain saturation with white noise [87,88], both processes are Markovian and characterized by the Lindblad master equation [89,90], where the gain and loss are modeled by constants $[14,20,51,91,92]$.

We consider an ideal case of resonators with negligible backscattering [93]. In the coupled-mode theory [94,95], the equation of motion for resonators $j \neq-1,0$, and $\alpha$ in the system illustrated in Fig. 1(a) is

$i \frac{d \psi_{j}}{d t}=\omega_{c} \psi_{j}-\psi_{j-1}-\psi_{j+1}$,

otherwise,

$i \frac{d \psi_{-1}}{d t}=\omega_{c} \psi_{-1}-\psi_{-2}-\psi_{0}-\psi_{\alpha}$,

$i \frac{d \psi_{\alpha}}{d t}=\left(\omega_{c}+V_{\alpha}\right) \psi_{\alpha}-\psi_{-1}-e^{-i \phi_{\alpha}} \psi_{0}$,

$i \frac{d \psi_{0}}{d t}=\omega_{c} \psi_{0}-\psi_{-1}-\psi_{1}-e^{i \phi_{\alpha}} \psi_{\alpha}$, (a)
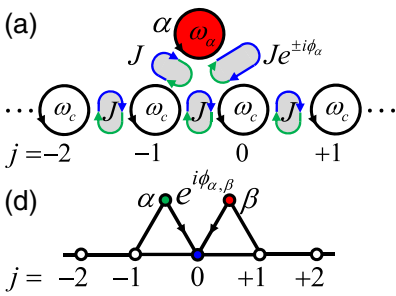

$\begin{array}{ll}\text { (b) } \phi_{\alpha}=\pi+k & \text { (c) } \phi_{\alpha}=\pi-k\end{array}$
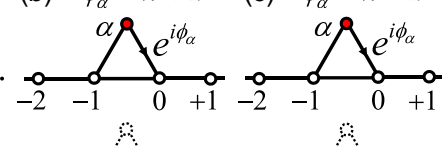

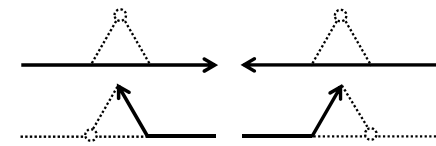

FIG. 1. (a) Schematic of uniform resonator chain with one sidecoupled resonator, $\omega_{\alpha}=\omega_{c}+V_{\alpha}$. The blue and green arrows indicate the optical path lengths for counterclockwise mode (black arrow) photons tunneling between resonators in opposite directions. (b) Right UPA: right perfect absorption and left resonant transmission. (c) Left UPA: left perfect absorption and right resonant transmission. The upper panels illustrate UPA configurations with opposite synthetic magnetic fluxes, $k$ is the incident wave vector, and $V_{\alpha}=-e^{i k}$. The central panels illustrate destructive interference at the side-coupled resonator $\alpha$ from one incident direction, and the lower panels illustrate perfect absorption from the opposite incident direction. The dotted line indicates the equivalently decoupled part. (d) Schematic of uniform resonator chain with two side-coupled resonators. $j$ is the resonator index, $\omega_{\alpha, 0, \beta}=\omega_{c}+V_{\alpha, 0, \beta}$. where $\psi_{j}=f_{j} e^{-i \omega t}$ is the field amplitude and $f_{j}$ is the steady-state wave function of resonator $j$ in the elastic scattering process with $f_{j}=A e^{i k j}+B e^{-i k j}(j<0)$ and $f_{j}=C e^{i k j}+D e^{-i k j}(j>0) . k \in[-\pi, \pi]$ is the dimensionless Bloch wave vector $[51,62,63,80]$. The resonator chain supported dispersion relation is $\omega=\omega_{c}-2 J \cos k[51,76]$; a resonant incidence with frequency $\omega_{c}$ has the wave vector $k=\pi / 2$.

Unidirectional destructive interference.-Synthetic magnetic flux affects the interference and breaks the system symmetry and the reciprocity of transmission [93]. When synthetic magnetic flux matches the incident wave vector $\phi_{\alpha}=\pi \pm k$, incidences from both sides are reflectionless. The side-coupled resonator $\alpha$ is equivalently isolated for the left (right) incidence because of the destructive interference induced by $\phi_{\alpha}=\pi+k\left(\phi_{\alpha}=\pi-k\right)$; the wave function at resonator $\alpha$ is zero. For the right (left) incidence, the wave function of resonator $\alpha$ does not vanish, it varies according to $V_{\alpha}$ and affects the right (left) transmission.

The reflection and transmission coefficients are $r_{L}=$ $B / A$ and $t_{L}=C / A$ for the left incidence $(D=0)$, and $r_{R}=C / D$ and $t_{R}=B / D$ for the right incidence $(A=0)$. The scattering matrix characterizes the relationship between input and output $[48,60]$

$$
\left(\begin{array}{l}
B \\
C
\end{array}\right)=S\left(\begin{array}{l}
A \\
D
\end{array}\right)
$$

Here, the scattering matrix is asymmetric [93]

$S=\left(\begin{array}{cc}r_{L} & t_{R} \\ t_{L} & r_{R}\end{array}\right)=\left(\begin{array}{cc}0 & \left(e^{i k}+V_{\alpha}\right) /\left(e^{-i k}+V_{\alpha}\right) \\ 1 & 0\end{array}\right)$,

induced by the asymmetric coupling. In Hermitian systems (real $V_{\alpha}$ ), the transmittivity is $\left|t_{L}\right|^{2}=\left|t_{R}\right|^{2}=1 ; f_{\alpha}$ vanishes for the left incidence, but is nonzero for the right incidence. In non-Hermitian systems (complex $V_{\alpha}$ ), the transmittivity is asymmetric, $\left|t_{L}\right|^{2} \neq\left|t_{R}\right|^{2}$.

At $\phi_{\alpha}=\pi+k$, the scattering of left incidence with wave vector $k$ is fixed even though $V_{\alpha}$ varies; the perfect absorption occurs for the right incidence when $V_{\alpha}=-e^{i k}$ [Fig. 1(b)]. In contrast to an isolator [60], the scattering matrix $S=\left(\begin{array}{ll}0 & 0 \\ 1 & 0\end{array}\right)$ is for the CCW mode and its transpose $S^{T}$ is for the CW mode [93]. The wave function for the right incidence consists of an incoming wave that is completely absorbed at resonator $\alpha$ without reflection, i.e., $f_{j}=0$ $(j<0), f_{j}=e^{-i k j}(j \geq 0)$, and $f_{\alpha}=-1$, with $r_{L}=r_{R}=$ $t_{R}=0$ and $\left|t_{L}\right|=1$, behaving similarly in a coupled helical waveguide design [80], but differently in the transmissionless UPA [52]. When the synthetic magnetic flux is opposite $\phi_{\alpha}=\pi-k$, the wave function is a left-right mirror reflection of that at $\phi_{\alpha}=\pi+k$, where resonator $\alpha$ is isolated for the right incidence [Fig. 1(c)]. A right UPA 
for the CCW mode is a left UPA for the CW mode, and UPA realizes chiral mode isolation [64-66].

UL occurs at $V_{\alpha}=-e^{-i k}$ when $t_{R}$ diverges $[38,51,96]$, where resonator $\alpha$ has an equal amount of gain in contrast to the UPA. The wave function for the right incidence consists of an outgoing wave that satisfies the boundary condition of lasing without any injection, i.e., $f_{j}=e^{-i k j}$ $(j<0), f_{j}=0(j \geq 0)$, and $f_{\alpha}=1$.

Incident direction independent wave propagation.-UDI facilitates the design of optical control devices, leading to an isolation of the side-coupled resonator in one incident direction and providing an opportunity to unidirectionally manipulate the waves. When assembling one more sidecoupled resonator, the cooperation between synthetic magnetic fluxes in UDIs enriches the intriguing asymmetric dynamics [82].

The equations of motion for the configuration of two side-coupled resonators [Fig. 1(d)] are Eqs. (1)-(3) and

$$
\begin{aligned}
& i \frac{d \psi_{0}}{d t}=\left(\omega_{c}+V_{0}\right) \psi_{0}-\psi_{-1}-\psi_{1}-e^{i \phi_{\alpha}} \psi_{\alpha}-e^{i \phi_{\beta}} \psi_{\beta}, \\
& i \frac{d \psi_{\beta}}{d t}=\left(\omega_{c}+V_{\beta}\right) \psi_{\beta}-\psi_{1}-e^{-i \phi_{\beta}} \psi_{0}, \\
& i \frac{d \psi_{1}}{d t}=\omega_{c} \psi_{1}-\psi_{0}-\psi_{2}-\psi_{\beta},
\end{aligned}
$$

where the real (imaginary) parts of $V_{\beta}$ and $V_{0}$ represent the frequency detunings (net gains or losses) of resonators $\beta$ and 0 , respectively.

At fixed synthetic magnetic fluxes $\phi_{\alpha}=\phi_{\beta}=\pi+k$, Figs. 2(a) and 2(b) illustrate the wave propagation for opposite incidences. In Fig. 2(a), the wave resonantly transmits at resonator $\alpha$ because of the destructive interference. Then, the transmitted wave is scattered at resonator 0 , and corresponding reflection and transmission
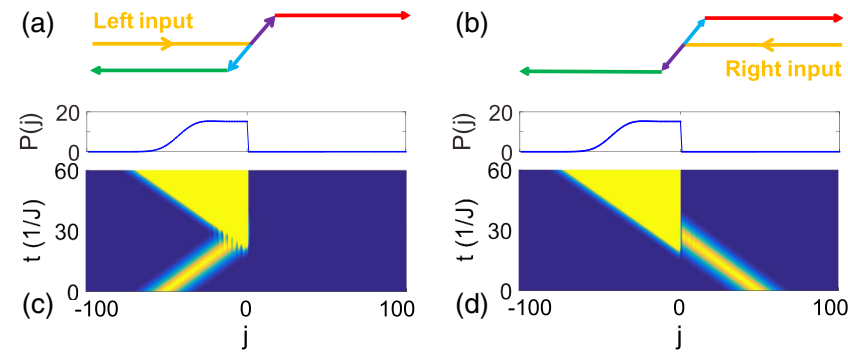

FIG. 2. (a), (b) Schematic of the incident direction independent wave propagation. (c), (d) Simulations of single-direction lasing at $V_{\alpha}=-e^{-i k}, V_{\beta}=-e^{i k}$ in Fig. 1(d). The Gaussian wave packet is $|\Psi(0, j)\rangle=(\sqrt{\pi} / \sigma)^{-1 / 2} \sum_{j} e^{-\left(\sigma^{2} / 2\right)\left(j-N_{c}\right)^{2}} e^{i k_{c} j}|j\rangle$, centered at $N_{c}$, where $k_{c}=\pi / 3$ is the wave vector, $\sigma=0.1$, and $j$ is the resonator index. The resonator chain is cut at $j= \pm 100$. The blue curves in (c), (d) depict the wave intensities $P(j)=|\Psi(t, j)|^{2}$ at time $t=60 / J$. Other parameters are $\phi_{\alpha}=\phi_{\beta}=\pi+k, V_{0}=-2 i \sin k$, and $k=\pi / 3$. coefficients are $r_{0}=V_{0} /\left(2 i \sin k-V_{0}\right)$ and $t_{0}=r_{0}+1$ [97]. The reflected portion (cyan arrow) passes through resonator $\alpha$ from the right side without reflection (green arrow) and forms the left reflection; the transmitted portion (purple arrow) passes through resonator $\beta$ from the left side without reflection (red arrow) and forms the left transmission. The green (red) arrow represents the reflectionless transmission modulated by resonator $\alpha(\beta)$. The reflection and transmission coefficients are [93]

$$
\begin{array}{ll}
r_{L}=r_{0} \frac{e^{i k}+V_{\alpha}}{e^{-i k}+V_{\alpha}}, & t_{L}=t_{0} \frac{e^{i k}+V_{\beta}}{e^{-i k}+V_{\beta}} ; \\
r_{R}=r_{0} \frac{e^{i k}+V_{\beta}}{e^{-i k}+V_{\beta}}, & t_{R}=t_{0} \frac{e^{i k}+V_{\alpha}}{e^{-i k}+V_{\alpha}} .
\end{array}
$$

The synthetic magnetic fluxes allow that the propagating waves in the left (right) chain after scattering are $V_{\beta(\alpha)}$ independent, regardless of the incident direction. This enables an incident direction independent wave propagation when $\left|r_{0}\right|=\left|t_{0}\right|$ (requiring $\left|2 \sin k / V_{0}\right|=1$ ): the left reflectivity and transmittivity equal to the right transmittivity and reflectivity, respectively.

$$
\left|r_{L}\right|^{2}=\left|t_{R}\right|^{2}, \quad\left|t_{L}\right|^{2}=\left|r_{R}\right|^{2} .
$$

The left- and right-going propagating wave intensities after scattering are identical for incidences impinging from both directions and are separately tuned by $V_{\alpha}$ and $V_{\beta}$, respectively. The wave impinging from either direction is equally divided at resonator 0 with $\left|r_{0}\right|=\left|t_{0}\right|=\sqrt{1 /(2-2 \sin \varphi)}$ when $V_{0}=2 e^{i \varphi} \sin k$.

The incident direction independent wave propagation occurs at

$$
\phi_{\alpha}=\phi_{\beta}=\pi+k, \quad V_{0}=2 e^{i \varphi} \sin k .
$$

Resonator $\beta(\alpha)$ being $V_{\beta(\alpha)}=-e^{i k}$ induces a perfect absorption of the right-(left-)going propagating wave, except that the system is at the SS when $V_{0}=2 i \sin k$, where lasing is bidirectional, or when $V_{\alpha(\beta)}=-e^{-i k}$, where the lasing is unidirectional toward the left (right) and the propagating wave in the right (left) chain vanishes after scattering, e.g., UL occurs when $V_{\alpha}=-e^{-i k}, V_{\beta}=-e^{i k}$, and $V_{0}=-2 i \sin k$. The lasing wave is emitted from the left chain, independent of the incident direction, and the right-going propagating wave vanishes, being absorbed at resonator $\beta$. The reflection and transmission coefficients satisfy

$$
\left|r_{L}\right|=\left|t_{R}\right| \rightarrow \infty, \quad t_{L}=r_{R}=0 .
$$

The wave function is $f_{j}=e^{-i k j}(j<0), f_{j}=0(j \geq 0)$, $f_{\alpha}=1$, and $f_{\beta}=0$, consisting of the outgoing wave in the 
left chain. For an incidence from either direction, the lasing wave is emitted in a single direction: leftward. The simulation of single-direction lasing is depicted in Figs. 2(c) and 2(d). A Gaussian wave packet excites the wave emission, characterized by a Gaussian error function and its intensity increases linearly [98]. At $V_{0}=2 i \sin k$, lasing is bidirectional when $V_{\alpha, \beta} \neq-e^{i k}$; the wave emission is symmetric if $V_{\alpha, \beta}$ is real, but asymmetric if complex $V_{\alpha} \neq V_{\beta}$. When $V_{\alpha(\beta)}=-e^{i k}$, the wave emission is absorbed at resonator $\alpha$ $(\beta)$ and the lasing becomes unidirectional with vanishing emission toward the left (right). When $V_{\alpha}=V_{\beta}=-e^{-i k}$, lasing is bidirectional and $V_{0}$ controls the asymmetry of lasing amplitude.

Another intriguing application is the one-way propagation at $V_{\alpha}=-\cos k+3^{ \pm 1} i \sin k, \quad V_{\beta}=-e^{i k}, \quad$ and $V_{0}=-2 i \sin k$, previously designed using different strategies [82-84], rectifying waves with $\left|r_{L}\right|=\left|t_{R}\right|=1$ and $t_{L}=r_{R}=0$.

For $V_{0}=0$, the scattering is both sides reflectionless when $V_{\alpha, \beta} \neq-e^{-i k}$. The left (right) transmission depends on $V_{\beta(\alpha)}$. When $V_{\alpha}=V_{\beta}=-e^{-i k}$, the transmission coefficients diverge with finite reflections. When $V_{\alpha}=V_{\beta}=-e^{i k}$, the system completely absorbs the incidence from either direction without reflection. When $V_{\alpha}=-e^{-i k}$ and $V_{\beta}=-e^{i k}$, UL occurs with $\left|r_{L}\right|=1$, $t_{L}=r_{R}=0$, and $\left|t_{R}\right| \rightarrow \infty$ (square in Fig. 3).

$\mathcal{P} \mathcal{T}$-symmetric side-coupled resonators.-UPA and UL form a Hermitian conjugation pair. Their SSs coincide and vanish at series connection of the two structures in a $\mathcal{P} \mathcal{T}$-symmetric manner. The wave emission is absorbed and leaves finite scattering intensities.

Figure 3 depicts $\left|r_{L, R}\right|$ and $\left|t_{L, R}\right|$ for $k=\pi / 3$ [93]. $\left|\phi_{\alpha(\beta)}\right|=2 \pi / 3$ produces a wave emission (absorption). $\left|r_{L}\right|=1$ at $\left|\phi_{\beta}\right|=2 \pi / 3$ and $\left|r_{L}\right| \rightarrow \infty$ at $\left|\phi_{\alpha}\right|=2 \pi / 3$ and $\left|\phi_{\beta}\right| \neq 2 \pi / 3$ [Fig. 3(a)]. $V_{\beta}=-e^{i k}$ results in $f_{0}=0$ for the right incidence except when $r_{L}$ diverges; consequently, $r_{R}=0$. Figures 3(b) and 3(c) depict $\left|t_{L}\right|$ and $\left|t_{R}\right|$. $\left|t_{L}\right|$ diverges at $\phi_{\alpha}=2 \pi / 3$, vanishes at $\phi_{\beta}=-2 \pi / 3$, and becomes unity at $\phi_{\alpha}=2 \pi / 3$ and $\phi_{\beta}=-2 \pi / 3$, where the

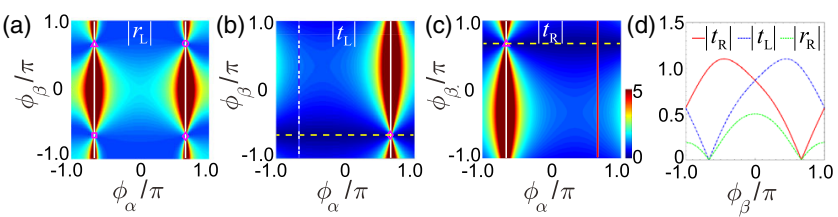

FIG. 3. Density plots of (a) $\left|r_{L}\right|$, (b) $\left|t_{L}\right|$, and (c) $\left|t_{R}\right|$ as functions of $\phi_{\alpha}$ and $\phi_{\beta} . r_{R}=0$ for $\left|\phi_{\alpha}\right| \neq 2 \pi / 3$. (d) $\left|t_{L}\right|\left(\left|t_{R}\right|\right)$ for $\phi_{\alpha}=-2 \pi / 3(2 \pi / 3)$ and $\left|r_{R}\right|$ at when $r_{L}$ diverges. Solid white (dashed yellow) lines indicate the divergence (zero) at the SS. SSs coincide with unity values at the marked points in (b), (c). Color bars are all in (c), $\left|r_{L}\right|$ and $\left|t_{L, R}\right|$ are cut to five. The parameters are $V_{\alpha}=-e^{-i \pi / 3}, V_{\beta}=-e^{i \pi / 3}, V_{0}=0$, and the incident wave vector is $k=\pi / 3$.
SSs of the side-coupled structures coincide. Figure 3(d) depicts the scattering coefficients at $\left|\phi_{\alpha}\right|=2 \pi / 3 \quad\left(r_{L}\right.$ divergence), implied by the dash-dotted blue line $\left[\left|t_{L}\right|\right.$ in Fig. 3(b)] and solid red line $\left[\left|t_{R}\right|\right.$ in Fig. 3(c)]. At $\phi_{\alpha}+\phi_{\beta}=0$, the system is $\mathcal{P} \mathcal{T}$ symmetric; the scattering coefficients converge when $\phi_{\alpha}=-\phi_{\beta}= \pm 2 \pi / 3 . \mathcal{P} \mathcal{T}$ symmetry ensures that the persistent wave emission from resonator $\alpha$ is directly absorbed at resonator $\beta$ and forms a unity transmittivity. The scattering coefficients satisfy $\left|r_{L}\right|=\left|t_{L}\right|=\left|t_{R}\right|=1,\left|r_{R}\right|=0$, and the SS vanishes. At $\phi_{\alpha}=\phi_{\beta}=2 \pi / 3$, a persistent right-going wave emission for the left incidence and a perfect absorption for the right incidence occur: $\left|r_{L}\right|=1,\left|t_{L}\right| \rightarrow \infty$, and $r_{R}=t_{R}=0$. At $\phi_{\alpha}=\phi_{\beta}=-2 \pi / 3$, a persistent left-going wave emission for the right incidence and a full reflection for the left incidence occur [93].

Simultaneous unidirectional lasing and perfect absorption.-UPA prevents the backward flow without affecting the forward propagation, which is a versatile building block for light manipulation. Series combination of several UPAs and ULs enables more intriguing asymmetric phenomena (Fig. 4).

Connecting a right UPA on the left side of the two sidecoupled resonators in the situation marked by the magenta circle in Fig. 3, the finite left reflection from the right two side-coupled resonators is perfectly absorbed; consequently, a reflectionless left incident unidirectional lasing and right incident perfect absorption [Figs. 4(a) and 4(b)] is achieved in the configuration of Fig. 4(c). If all the synthetic magnetic fluxes in Fig. 4(c) are opposite, which correspond to the configuration experienced by the $\mathrm{CW}$ mode [Fig. 4(f)], the dynamics switch between the left and right incidences in contrast to the CCW mode [Figs. 4(d) and 4(e)]. Unidirectional lasing of different modes are toward opposite directions. Simultaneously exciting the $\mathrm{CCW}$ and $\mathrm{CW}$ modes in the left (right) side, the CCW (CW) mode induces a unidirectional lasing toward the right (left) side and the CW (CCW) mode is perfectly absorbed.
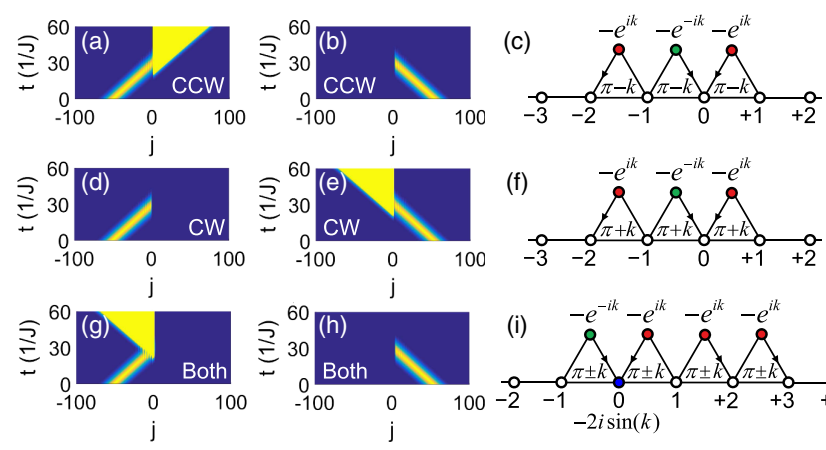

FIG. 4. Snapshots of the wave intensities and schematics of the equivalent systems for (a)-(f) transmissionless and (g)-(i) reflectionless unidirectional lasing and perfect absorption for a Gaussian wave packet of $\sigma=0.1, k_{c}=\pi / 3$. The arrows indicate the phase directions with values inside, $k=\pi / 3$. 
Connecting two right UPAs on the right side of a singledirection lasing [Figs. 2(c) and 2(d)] to form the configuration shown in Fig. 4(i), the right incidence is perfectly absorbed before inducing a unidirectional lasing, resulting in a transmissionless left incident unidirectional lasing and right incident perfect absorption [Figs. 4(g) and 4(h)]. The $\mathrm{CW}$ mode experiences opposite synthetic magnetic fluxes, a left incident bidirectional lasing and right incident perfect absorption is realized by the left two side-coupled resonators [93] and the right two right UPAs change into left UPAs, which perfectly absorb the right-going lasing. Notably, both the CCW and CW modes possess identical dynamical phenomena.

Discussion.-In summary, the bidirectional reflectionlessness as a desirable feature of UDI allows scalable combination of unidirectional dynamics, and properly assembling distinct UDIs enriches the unidirectionality. The incident direction independent wave propagation is proposed, including the unidirectional lasing toward the single definite direction and the one-way propagation. The simultaneous unidirectional lasing and perfect absorption is allowed. Our findings are applicable in optical waveguides [80].

The imperfections in the resonator as defects and surface roughness result in backscattering [99-101], which induces a mode coupling and mixes the CCW and CW modes [99]. The backscattering results in mode interchanging and is unfavorable for the desirable unidirectional functionalities [102]. Directional couplers can be used to reduce the influence of backscattering [103]. As shown in the Supplemental Material [93], UPA absorbs one mode without affecting the other mode, which helps prevent the unwanted backscattering induced mode accumulation in one side; moreover, the performances of UPA, singledirection lasing, and transmissionless unidirectional lasing and perfect absorption remain good at weak backscattering. Alternatively, the backscattering is a useful resource for optical sensing [28,29,31].

Synthetic magnetic flux has been realized in quantum regime $[58,79,104]$, and it would be interesting to investigate the unidirectionality in quantum dots, cold atoms, or trapped ions in the frameworks of non-Hermitian physics and chiral quantum optics [55-59]. Our findings open up new directions for designing novel lasers and optical control devices including but not limited to laser, absorber, rectifier, isolator, and modulator in a variety of areas in optics and beyond.

This work was supported by NSFC (Grant No. 11605094) and Tianjin Natural Science Foundation (Grant No. 16JCYBJC40800).

*jinliang@nankai.edu.cn

[1] C. M. Bender and S. Boettcher, Phys. Rev. Lett. 80, 5243 (1998).
[2] A. Mostafazadeh, J. Math. Phys. 43, 205 (2002).

[3] H. F. Jones, J. Phys. A 38, 1741 (2005).

[4] A. Ruschhaupt, F. Delgado, and J. G. Muga, J. Phys. A 38, L171 (2005).

[5] R. El-Ganainy, K. G. Makris, D. N. Christodoulides, and Z. H. Musslimani, Opt. Lett. 32, 2632 (2007).

[6] Z. H. Musslimani, K. G. Makris, R. El-Ganainy, and D. N. Christodoulides, Phys. Rev. Lett. 100, 030402 (2008).

[7] S. Klaiman, U. Günther, and N. Moiseyev, Phys. Rev. Lett. 101, 080402 (2008).

[8] L. Jin and Z. Song, Phys. Rev. A 80, 052107 (2009).

[9] Z. Lin, H. Ramezani, T. Eichelkraut, T. Kottos, H. Cao, and D. N. Christodoulides, Phys. Rev. Lett. 106, 213901 (2011).

[10] M. Liertzer, L. Ge, A. Cerjan, A. D. Stone, H. E. Türeci, and S. Rotter, Phys. Rev. Lett. 108, 173901 (2012).

[11] D. A. Zezyulin and V. V. Konotop, Phys. Rev. Lett. 108, 213906 (2012).

[12] L. Ge and A. D. Stone, Phys. Rev. X 4, 031011 (2014).

[13] X. Zhu, H. Ramezani, C. Shi, J. Zhu, and X. Zhang, Phys. Rev. X 4, 031042 (2014).

[14] L. Chang, X. Jiang, S. Hua, C. Yang, J. Wen, L. Jiang, G. Li, G. Wang, and M. Xiao, Nat. Photonics 8, 524 (2014).

[15] H. Jing, S. K. Özdemir, X.-Y. Lü, J. Zhang, L. Yang, and F. Nori, Phys. Rev. Lett. 113, 053604 (2014).

[16] R. Fleury, D. Sounas, and A. Alù, Nat. Commun. 6, 5905 (2015).

[17] S. V. Suchkov, F. Fotsa-Ngaffo, A. Kenfack-Jiotsa, A. D Tikeng, T. C Kofane, Y. S Kivshar, and A. A Sukhorukov, New J. Phys. 18, 065005 (2016).

[18] A. Guo, G. J. Salamo, D. Duchesne, R. Morandotti, M. Volatier-Ravat, V. Aimez, G. A. Siviloglou, and D. N. Christodoulides, Phys. Rev. Lett. 103, 093902 (2009).

[19] C. E. Rüter, K. G. Makris, R. El-Ganainy, D. N. Christodoulides, M. Segev, and D. Kip, Nat. Phys. 6, 192 (2010).

[20] B. Peng, S. K. Özdemir, F. Lei, F. Monifi, M. Gianfreda, G. L. Long, S. Fan, F. Nori, C. M. Bender, and L. Yang, Nat. Phys. 10, 394 (2014).

[21] Z. Zhang, Y. Zhang, J. Sheng, L. Yang, M.-A. Miri, D. N. Christodoulides, B. He, Y. Zhang, and M. Xiao, Phys. Rev. Lett. 117, 123601 (2016).

[22] R. El-Ganainy, K. G. Makris, M. Khajavikhan, Z. H. Musslimani, S. Rotter, and D. N. Christodoulides, Nat. Phys. 14, 11 (2018).

[23] C. Dembowski, B. Dietz, H.-D. Gräf, H. L. Harney, A. Heine, W. D. Heiss, and A. Richter, Phys. Rev. E 69, 056216 (2004).

[24] H. Cartarius, J. Main, and G. Wunner, Phys. Rev. Lett. 99, 173003 (2007).

[25] M. Müller and I. Rotter, J. Phys. A 41, 244018 (2008).

[26] R. Uzdin, A. Mailybaev, and N. Moiseyev, J. Phys. A 44, 435302 (2011).

[27] W. D. Heiss, J. Phys. A 45, 444016 (2012).

[28] J. Wiersig, Phys. Rev. Lett. 112, 203901 (2014).

[29] J. Wiersig, Phys. Rev. A 93, 033809 (2016).

[30] Z.-P. Liu, J. Zhang, S. K. Özdemir, B. Peng, H. Jing, X.-Y. Lü, C.-W. Li, L. Yang, F. Nori, and Y. X. Liu, Phys. Rev. Lett. 117, 110802 (2016).

[31] W. Chen, S. K. Özdemir, G. Zhao, J. Wiersig, and L. Yang, Nature (London) 548, 192 (2017). 
[32] H. Hodaei, A. U. Hassan, S. Wittek, H. Garcia-Gracia, R. El-Ganainy, D. N. Christodoulides, and M. Khajavikhan, Nature (London) 548, 187 (2017).

[33] B. Zhen, C. W. Hsu, Y. Igarashi, L. Lu, I. Kaminer, A. Pick, S.-L. Chua, J. D. Joannopoulos, and M. Soljačić, Nature (London) 525, 354 (2015).

[34] H. Menke, M. Klett, H. Cartarius, J. Main, and G. Wunner, Phys. Rev. A 93, 013401 (2016).

[35] J. Doppler, A. A. Mailybaev, J. Böhm, U. Kuhl, A. Girschik, F. Libisch, T. J. Milburn, P. Rabl, N. Moiseyev, and S. Rotter, Nature (London) 537, 76 (2016).

[36] H. Xu, D. Mason, L. Jiang, and J. G. E. Harris, Nature (London) 537, 80 (2016).

[37] K. Ding, G. Ma, M. Xiao, Z. Q. Zhang, and C. T. Chan, Phys. Rev. X 6, 021007 (2016).

[38] A. Mostafazadeh, Phys. Rev. Lett. 102, 220402 (2009).

[39] A. Mostafazadeh, Phys. Rev. Lett. 110, 260402 (2013).

[40] Y. D. Chong, L. Ge, H. Cao, and A. D. Stone, Phys. Rev. Lett. 105, 053901 (2010).

[41] S. Longhi, Phys. Rev. A 82, 031801(R) (2010).

[42] Y. D. Chong, L. Ge, and A. D. Stone, Phys. Rev. Lett. 106, 093902 (2011).

[43] W. Wan, Y. Chong, L. Ge, H. Noh, A. D. Stone, and H. Cao, Science 331, 889 (2011).

[44] Y. Sun, W. Tan, H.-Q. Li, J. Li, and H. Chen, Phys. Rev. Lett. 112, 143903 (2014).

[45] Z. J. Wong, Y.-L. Xu, J. Kim, K. O’Brien, Y. Wang, L. Feng, and X. Zhang, Nat. Photonics 10, 796 (2016).

[46] C. Hang, G. Huang, and V. V. Konotop, New J. Phys. 18, 085003 (2016).

[47] L. Ge and L. Feng, Phys. Rev. A 95, 013813 (2017).

[48] D. G. Baranov, A. Krasnok, T. Shegai, A. Alù, and Y. Chong, Nat. Rev. Mater. 2, 17064 (2017).

[49] A. Regensburger, C. Bersch, M.-A. Miri, G. Onishchukov, D. N. Christodoulides, and U. Peschel, Nature (London) 488, 167 (2012).

[50] L. Feng, Y.-L. Xu, W. S. Fegadolli, M.-H. Lu, J. E. B. Oliveira, V. R. Almeida, Y.-F. Chen, and A. Scherer, Nat. Mater. 12, 108 (2013).

[51] H. Ramezani, H.-K. Li, Y. Wang, and X. Zhang, Phys. Rev. Lett. 113, 263905 (2014).

[52] H. Ramezani, Y. Wang, E. Yablonovitch, and X. Zhang, IEEE J. Sel. Top. Quantum Electron. 22, 115 (2016).

[53] L. Jin, X. Z. Zhang, G. Zhang, and Z. Song, Sci. Rep. 6, 20976 (2016).

[54] B. Peng, S. K. Özdemir, M. Liertzer, W. Chen, J. Kramer, H. Yilmaz, J. Wiersig, S. Rotter, and L. Yang, Proc. Natl. Acad. Sci. U.S.A. 113, 6845 (2016).

[55] T. Ramos, H. Pichler, A. J. Daley, and P. Zoller, Phys. Rev. Lett. 113, 237203 (2014).

[56] H. Pichler, T. Ramos, A. J. Daley, and P. Zoller, Phys. Rev. A 91, 042116 (2015).

[57] I. Söllner, S. Mahmoodian, S. L. Hansen, L. Midolo, A. Javadi, G. Kiršanskè, T. Pregnolato, H. El-Ella, E. H. Lee, J. D. Song, S. Stobbe, and P. Lodahl, Nat. Nanotechnol. 10, 775 (2015).

[58] B. Vermersch, T. Ramos, P. Hauke, and P. Zoller, Phys. Rev. A 93, 063830 (2016).
[59] P. Lodahl, S. Mahmoodian, S. Stobbe, A. Rauschenbeutel, P. Schneeweiss, J. Volz, H. Pichler, and P. Zoller, Nature (London) 541, 473 (2017).

[60] D. Jalas, A. Petrov, M. Eich, W. Freude, S. Fan, Z. Yu, R. Baets, M. Popović, A. Melloni, J. D. Joannopoulos, M. Vanwolleghem, C. R. Doerr, and H. Renner, Nat. Photonics 7, 579 (2013).

[61] X. Yin and X. Zhang, Nat. Mater. 12, 175 (2013).

[62] J. G. Muga, J. P. Palao, B. Navarro, and I. L. Egusquiza, Phys. Rep. 395, 357 (2004).

[63] F. Cannata, J.-P. Dedonder, and A. Ventura, Ann. Phys. (Amsterdam) 322, 397 (2007).

[64] L. Bi, J. Hu, P. Jiang, D. H. Kim, G. F. Dionne, L. C. Kimerling, and C. A. Ross, Nat. Photonics 5, 758 (2011).

[65] L. Fan, J. Wang, L. T. Varghese, H. Shen, B. Niu, Y. Xuan, A. M. Weiner, and M. Qi, Science 335, 447 (2012).

[66] Z. Yu and S. Fan, Nat. Photonics 3, 91 (2009).

[67] D.-W. Wang, H.-T. Zhou, M.-J. Guo, J.-X. Zhang, J. Evers, and S.-Y. Zhu, Phys. Rev. Lett. 110, 093901 (2013).

[68] I. Shomroni, S. Rosenblum, Y. Lovsky, O. Bechler, G. Guendelman, and B. Dayan, Science 345, 903 (2014).

[69] S. Rosenblum, O. Bechler, I. Shomroni, Y. Lovsky, G. Guendelman, and B. Dayan, Nat. Photonics 10, 19 (2016).

[70] R. Fleury, D. L. Sounas, C. F. Sieck, M. R. Haberman, and A. Alù, Science 343, 516 (2014).

[71] K. Fang, Z. Yu, and S. Fan, Nat. Photonics 6, 782 (2012).

[72] L. D. Tzuang, K. Fang, P. Nussenzveig, S. Fan, and M. Lipson, Nat. Photonics 8, 701 (2014).

[73] L. Lu, J. D. Joannopoulos, and M. Soljačić, Nat. Photonics 8, 821 (2014).

[74] E. Li, B. J. Eggleton, K. Fang, and S. Fan, Nat. Commun. 5, 3225 (2014).

[75] M. Hafezi, E. A. Demler, M. D. Lukin, and J. M. Taylor, Nat. Phys. 7, 907 (2011).

[76] M. Hafezi, Phys. Rev. Lett. 112, 210405 (2014).

[77] M. Hafezi, Int. J. Mod. Phys. B 28, 1441002 (2014).

[78] S. Longhi, Opt. Lett. 39, 5892 (2014).

[79] P. Roushan et al., Nat. Phys. 13, 146 (2017).

[80] S. Longhi, Opt. Lett. 40, 1278 (2015).

[81] X. Q. Li, X. Z. Zhang, G. Zhang, and Z. Song, Phys. Rev. A 91, 032101 (2015).

[82] L. Jin, P. Wang, and Z. Song, New J. Phys. 19, 015010 (2017).

[83] S. Longhi, D. Gatti, and G. Della Valle, Phys. Rev. B 92, 094204 (2015).

[84] S. Lepri and G. Casati, Phys. Rev. Lett. 106, 164101 (2011).

[85] J. D'Ambroise, P. G. Kevrekidis, and S. Lepri, J. Phys. A 45, 444012 (2012).

[86] V. V. Konotop, J. Yang, and D. A. Zezyulin, Rev. Mod. Phys. 88, 035002 (2016).

[87] H. Haken, Laser Theory (Springer, Berlin, 1970).

[88] R. Roy, A. W. Yu, and S. Zhu, Phys. Rev. Lett. 55, 2794 (1985).

[89] H. P. Breuer and F. Petruccione, The Theory of Open Quantum Systems (Oxford University Press, New York, 2007).

[90] M. O. Scully and M. S. Zubairy, Quantum Optics (Cambridge University Press, Cambridge, England, 1997). 
[91] L. Feng, Z. J. Wong, R.-M. Ma, Y. Wang, and X. Zhang, Science 346, 972 (2014).

[92] L. Jin, M. Pfender, N, Aslam, P. Neumann, S. Yang, J. Wrachtrup, and R.-B. Liu, Nat. Commun. 6, 8251 (2015).

[93] See Supplemental Material at http://link.aps.org/ supplemental/10.1103/PhysRevLett.121.073901 for the details on the derivation of scattering coefficients, discussions on the CCW and CW modes, and the influence of backscattering on the asymmetric dynamics.

[94] H. A. Haus and W. P. Huang, Proc. IEEE 79, 1505 (1991).

[95] J. D. Joannopoulos, S. G. Johnson, J. N. Winn, and R. D. Meade, Photonic Crystals: Molding the Flow of Light (Princeton University Press, Princeton, NJ, 2008).

[96] H. Ramezani, S. Kalish, I. Vitebskiy, and T. Kottos, Phys. Rev. Lett. 112, 043904 (2014).
[97] W. Kim, L. Covaci, and F. Marsiglio, Phys. Rev. B 74, 205120 (2006).

[98] P. Wang, L. Jin, G. Zhang, and Z. Song, Phys. Rev. A 94, 053834 (2016).

[99] T. J. Kippenberg, S. M. Spillane, and K. J. Vahala, Opt. Lett. 27, 1669 (2002).

[100] M. Borselli, T. J. Johnson, and O. Painter, Opt. Express 13, 1515 (2005).

[101] F. Morichetti, A. Canciamilla, C. Ferrari, M. Torregiani, A. Melloni, and M. Martinelli, Phys. Rev. Lett. 104, 033902 (2010).

[102] L. Jin and Z. Song, Phys. Rev. A 84, 042116 (2011).

[103] M. Hafezi, S. Mittal, J. Fan, A. Migdall, and J. M. Taylor, Nat. Photonics 7, 1001 (2013).

[104] N. Goldman, J. Dalibard, A. Dauphin, F. Gerbier, M. Lewenstein, P. Zoller, and I. B. Spielman, Proc. Natl. Acad. Sci. U.S.A. 110, 6736 (2013). 